\title{
Lacunarity as a texture measure for SAR imagery
}

\author{
G. M. HENEBRY $\dagger$ and H. J. H. KUX \\ DSM, Instituto Nacional de Pesquisas Espaciais (INPE), C.P. 515, \\ 12201-970 São José dos Campos, SP, Brazil
}

(Received 20 May 1994; in final form 12 September 1994)

\begin{abstract}
Lacunarity analysis is a simple technique for characterizing texture in binary images. Lacunarity quantifies deviation from translational invariance by describing the distribution of gaps within the image at multiple scales: the more lacunar an image, the more heterogeneous the spatial arrangement of gaps. For grey-level data, a series of binary images are formed through slicing the image histogram by quantiles. Characteristic decays of lacunarity as a function of window size permit scene object texture to be distinguished from speckle. Using a series of ERS-1 SAR images of the Brazilian Pantanal, we demonstrate how lacunarity functions can link image phenomenology with scene dynamics.
\end{abstract}

\section{Introduction}

The use of Synthetic Aperture Radar (SAR) imagery for environmental monitoring will increase during the decade as data from spaceborne platforms (ERS-1 and 2, Fuyo-1, Radarsat) become more readily available. Scene models (sensu Strahler $e$ t al. 1986) for radar remote sensing have focused primarily on descriptions of backscattering from static scene objects (e.g., Ulaby et al. 1990, Karam et al. 1992). The dynamic nature of environmental monitoring, however, necessitates the developIment of spatio-temporal scene models that describe image phenomenology and permit linkages to other dataforms, e.g., GIS, simulation models, optical imagery (Henebry 1993).

Texture analysis provides a first step toward developing dynamic scene models. Several studies have investigated the efficacy of texture measures in SAR image segmentation and classification using either autocorrelation/autocovariance formulae derived from simple phenomenological models of backscattering (e.g., Sheen and Johnston 1992; Rignot and Kwok 1993) or measures based on the Grey Level Cooccurrence Matrix (GLCM) (e.g., Ulaby et al. 1986, Barber and LeDrew 1991). The GLCM describes probabilities of the co-occurrence of two specific grey-levels given specific pixel locations in terms of relative direction and distance (Haralick and Shapiro 1992). Both approaches describe texture in terms of (dis)similarity based on spatial proximity (lag-neighbourhood) but they do not include an explicit consideration of scaling effects on spatial pattern. Further, GLCM measures do not explicitly address speckle noise, thus biasing estimates of scene texture.

We demonstrate a simple multi-scale technique, lacunarity analysis, that can distinguish between speckle-dominated texture and intrinsic scene texture. More significantly, lacunarity is a straightforward metric that facilitates the connection

†Permanent address: Division of Biology, Kansas State University, Manhattan, KS, 66506, U.S.A. 
between image phenomenology and scene dynamics. We demonstrate the technique on multi-temporal SAR imagery of the Pantanal, the largest wetland ecosystem on the planet.

\section{Methods}

\subsection{Lacunarity as a texture measure}

Lacunarity describes the complex intermingling of the shape and distribution of gaps within a binary image: a highly lacunar image exhibits gaps distributed across a broad range of sizes (Mandelbrot 1983, Plotnick et al. 1993). Lacunarity is an aspect of fractal geometry: lacunarity $(L)$ is the multiplicative prefactor in the general power-law formula of which the fractal dimension is the exponent, $F(x)=L x^{(D-E)}$. As a texture measure, lacunarity quantifies the deviation of a geometric object (e.g., shape, pattern, fractal) from translational invariance, an aspect of spatial stationarity (Plotnick et al. 1993). A simple estimate of lacunarity, the index $\Lambda(w)$, is the ratio of the variance of weighted number of occupied sites at observation window size $w$ to the square of the mean weighted number of sites plus 1 :

$$
\Lambda(w)=1+\operatorname{var}(w) / \operatorname{mean}^{2}(w)
$$

The maximum value of $\Lambda(w)$ occurs when the window size equals the spatial resolution of the image; it then equals the inverse of the proportion of occupied pixels. Conversely, the minimum value $(=1)$ occurs when the observation window equals the image size, i.e., when the variance is zero.

Calculating the lacunarity index across a series of window sizes and plotting the logarithm of the index against the logarithm of the window size, the resulting lacunarity function illustrates the scale-dependency of spatial nonstationarity. The decay pattern of the lacunarity function contains significant information about the spatial structure of the binary image (Plotnick et al. 1993). A spatially random image exhibits a swift decay to the minimum value. An image with self-similarity across some range of scales exhibits a linear decay, the slope of which is an estimate of the fractal dimension of the pattern within that range. For an image with an arrangement of objects at a particular scale, the lacunarity decay is slow until the window size exceeds the scale of the objects and is rapid thereafter. By varying window shape as well as size, lacunarity functions can also identify departures from another aspect of spatial stationarity: rotational invariance or isotropy.

\subsection{Study site}

The Pantanal is the largest wetland habitat on the planet: an immense alluvial plain formed during the Holocene, it covers $139000 \mathrm{~km}^{2}$ in Brazilian states of Mato Grosso and Mato Grosso do Sul and extends into Bolivia and Paraguay (Rizzini et al. 1988). The Pantanal is also one of the more radiometrically dynamic landscapes on the planet due to extensive seasonal flooding by the Rio Paraguai and its tributaries and by intra-basin precipitation. An ecotonal landscape, the Pantanal is a complex mosaic of shallow lakes, periodically inundated grasslands, and islands and elevated corridors of forest, which together supportan abundant and diverse fauna of birds, fish, reptiles, and mammals, including a few million head of cattle (Alho et al. 1988).

For this study we used ERS-1 images of the Nhecolândia region of the Pantanal (Table 1). This area comprises the southern tier of the vast $\left(\approx 50000 \mathrm{~km}^{2}\right)$ alluvial fan associated with the Rio Taquari and is characterized by hundreds of shallow 
Table 1. Acquisition data for ERS-1 SAR images.

\begin{tabular}{lccc}
\hline Date & $\begin{array}{c}\text { GMT } \\
\text { (hh:mm:ss) }\end{array}$ & $\begin{array}{c}\text { Lat./Long. of image centre } \\
\text { (ddd:mm:ss) }\end{array}$ & Orbit/Frame \\
\hline 12 December, 1992 & $13: 50: 14$ & S19:12:04/E304:02:06 & $7369 / 210$ \\
20 February, 1993 & $13: 50: 23$ & S19:12:40/E304:01:26 & $8371 / 210$ \\
1 May, 1993 & $13: 50: 12$ & S19:15:00/E304:01:19 & $9373 / 210$ \\
\hline
\end{tabular}

lakes, both freshwater (baias) and brackish (salinas). The December image represents the drier phase of the region's annual flooding cycle: lakes and islands, beaches and corridors are distinct. By February flooding had commenced but was not yet generalized: previously sharp edges are blurred. The May image represents the flooding peak: only the salinas retain a low backscattering; areas of inundation exhibit radiometric changes due to widespread growth of aquatic macrophytes and perennial grasses that emerged in the freshwater.

\subsection{Image registration, binary image formation, and lacunarity calculation}

We used georeferenced, ground-range projected, real-valued, 3-look SAR digital imagery processed by the INPE. The nominal ground resolution of these data were $25 \mathrm{~m}$ with a pixel spacing of $12.5 \mathrm{~m}$ in both range and azimuth. A 1024 line by 1024 pixel subimage was extracted from the central region of each image. The February and May data were registered to the December image with the Khoros warpimage program, which uses a least-squares fit of tiepoints to produce four reference points followed by a quadratic polynomial fit and bilinear interpolation (The Khoros Group, 1991). Keying on persistent lake features and islands, 28 and 15 tiepoints were used for February and May images, respectively. The resulting images were cropped to yield a common $590.24 \mathrm{~km}^{2}$ area ( 992 lines by 952 pixels) for analysis.

For each image, the quartiles of the frequency distribution $(\mathrm{Q} 1, \mathrm{Q} 2, \mathrm{Q} 3, \mathrm{Q} 4)$ were calculated and four binary images were formed by threshold filtering (figure 1). Lacunarity was estimated using square windows ranging from 1 to 32 pixels $(25 \mathrm{~m}$ to $800 \mathrm{~m}$ ) with 1000 random samples at each size. The lacunarity index was calculated at each window size by, 1, tabulating the frequency of occupied pixels per sample into size classes, 2 , normalizing the frequency counts by sample number to yield size class probabilities, 3 , weighting each size class probability by multiplying by its respective number of occupied pixels, 4 , calculating the mean and variance of the weighted probability distribution, and 5, calculating the index using (1).

\section{Results}

Comparison of lacunarity functions across the quartiles of a single image reveals that more spatial structure, in terms of the scale-dependency of translational invariance, is found in the tails of the image histogram (i.e., Q1 and Q4) than in the middle 50 per cent (figure 2). The smoother, more rapid decays of the Q2 and Q3 lacunarity functions contrast with the structured slower decays of Q1 and Q4.

Quartile-specific lacunarity functions exhibit temporal shifts in decay pattern. The Q1 lacunarity function is most strongly affected (figure $2(a)$ ). The slow decays of December and February indicate large patches ( $>64 \mathrm{ha}$ ) of comparable backscattering objects. In contrast, the swift quasi-linear decay of May suggests spatial self- 


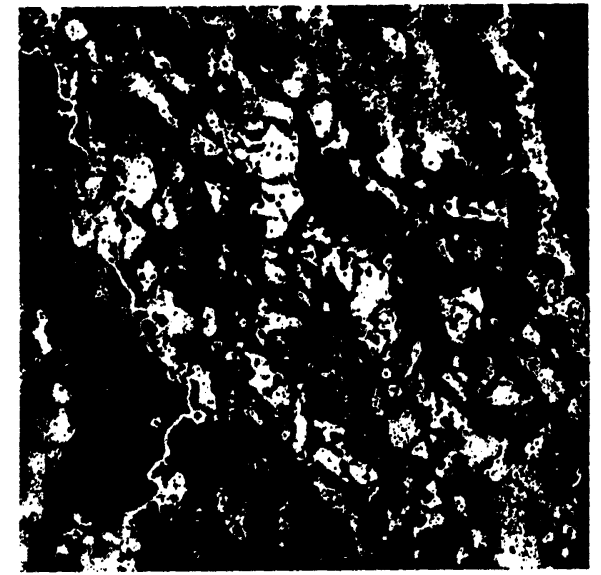

(a)

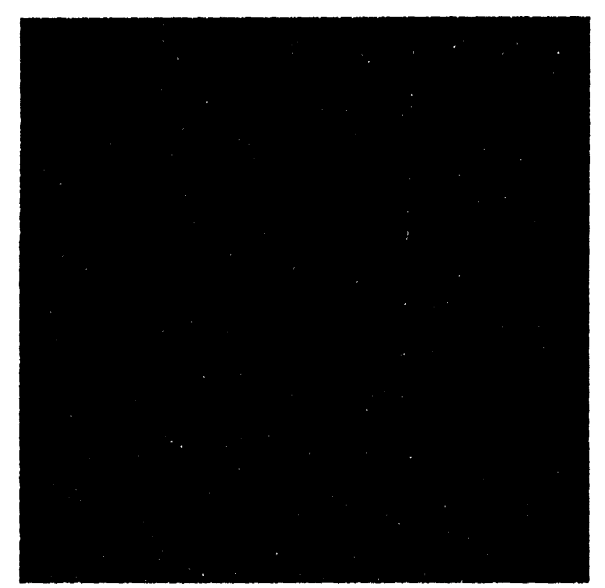

(c)

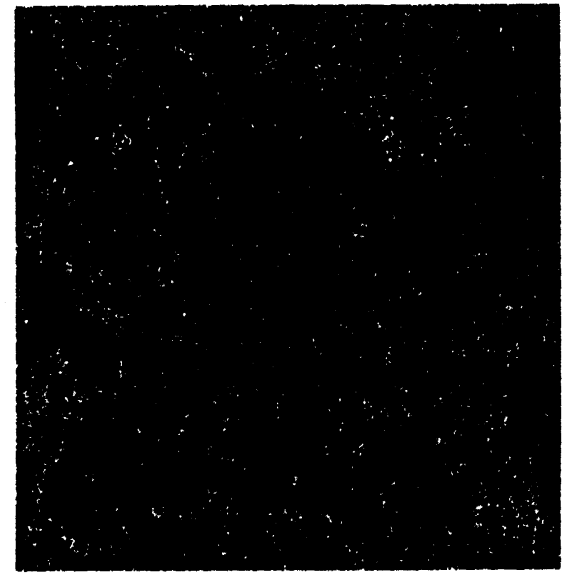

(b)

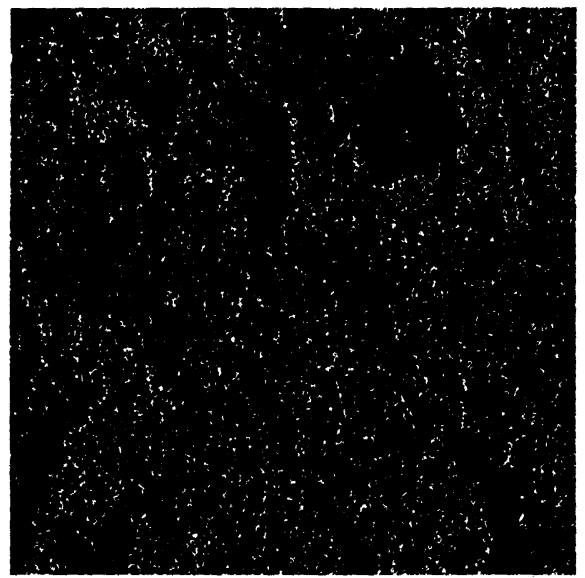

(d)

Figure 1. Binary images formed by the 12 December, 1992 ERS-1 SAR image from the Nhecolândia region of the Pantanal, Brazil: $(a)$ first quartile (Q1), (b) second quartile (Q2), (c) third quartile (Q3), and (d) fourth quartile (Q4).

similarity in the distribution of backscattering patches between 0.25 and 6.25 ha. Temporal changes in lacunarity for the other quartiles are less pronounced but a pattern of decreasing lacunarity (increasing translational invariance) from December to May is evident (figure $2(b, c, d)$ ).

\section{Discussion}

The temporal shifts observed in the lacunarity functions can be understood in terms of changes of spatial structure and scattering geometry in the scene being imaged. The Q1 binary image (figure $1(a)$ ) captures the spatial arrangement of those scene objects with low radar backscattering, predominantly areas of open water. The dramatic shift of the Q1 lacunarity function (figure $2(a)$ ) arises from a fundamental 

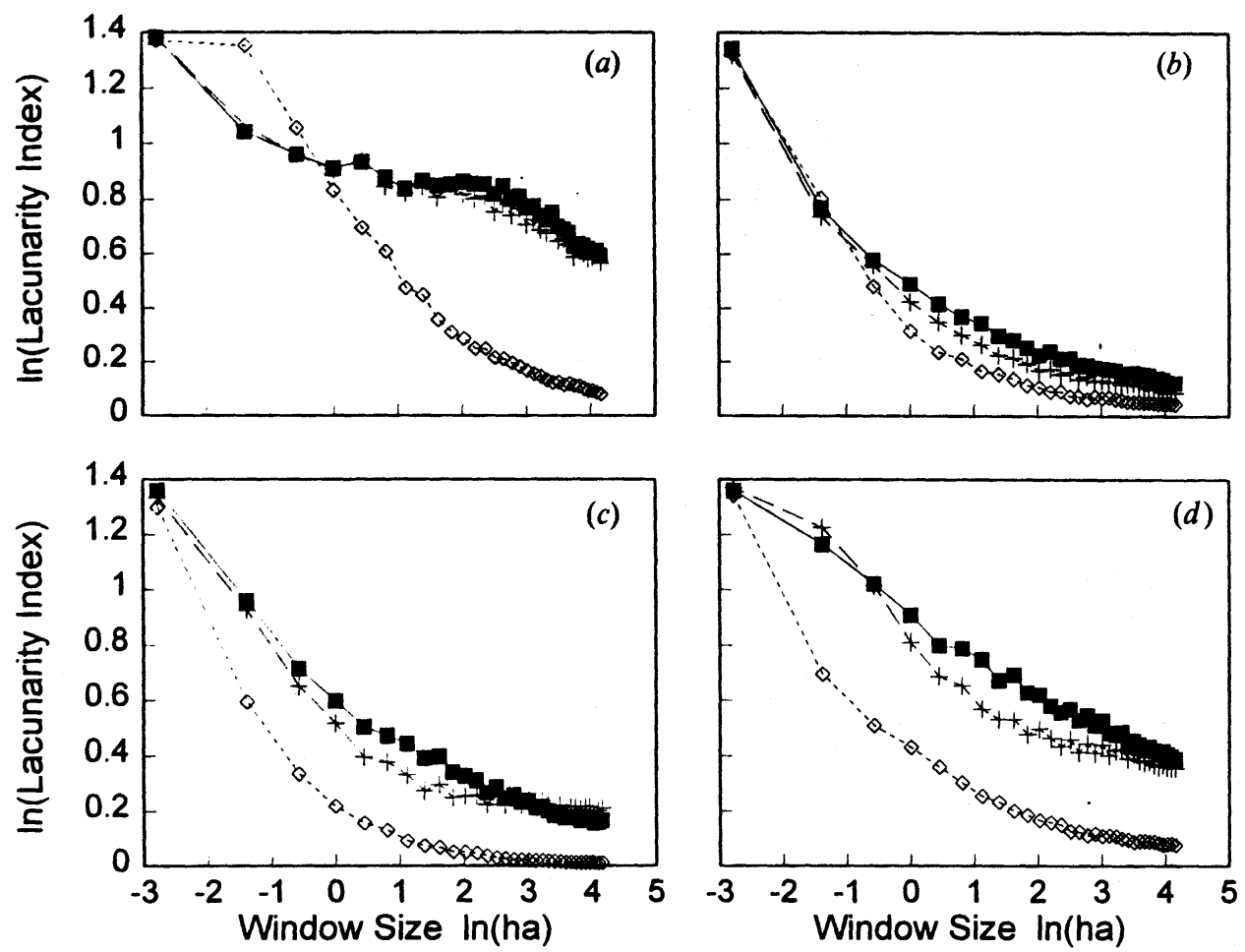

Figure 2. Lacunarity functions by quartile for images at three successive dates representing progressively higher water levels within the scene: $(a) \mathrm{Q} 1,(b) \mathrm{Q} 2,(c) \mathrm{Q} 3$, and $(d) \mathrm{Q} 4$. Square with solid line is 12 December, 1992. Cross with dashed line is 20 February 1993. Diamond with dotted line is 1 May, 1993.

change in the scene. By May the baias have significant canopies of emergent aquatic macrophytes and perennial grasses that increase the surface roughness and blur once sharp transitions from open water to sandy beaches with sparse vegetation and forested ridges.

Three arrangements are evident in the December Q2 image (figure 1(b)): 1. linear features corresponding to borders along open water; 2. large but diffuse clusters representing wetter vegetated areas; and 3. low-valued speckle noise randomly distributed through areas of dense woody vegetation. In contrast, the consistently rapid decays for Q2 (figure $2(b)$ ) suggest a spatially near-random landscape that becomes more homogenized through time. The Q2 scene is indeed dominated spatially by speckle: linear features are not well detected by a square window and diffuse clusters are ill-represented by method's density counting.

Only two features are evident in the December Q3 image (figure $1(c)$ ): continuous patches of wetter regions (black) and speckle noise within areas of high aboveground biomass (diffuse white). The Q3 lacunarity functions (figure $2(c)$ ) for December and February exhibit comparable decays with initial self-similarity shifting to an almost constant non-zero level. The May decay rapidly levels off near zero indicating a homogeneously random pattern of Q3 backscatterers in response to inundation. 
The Q4 binary image contains the pixels exhibiting the highest degree of backscattering within the scene, such as those associated with the forested corridors and islands. The December Q4 image (figure 1(d)), although similar to Q3, shows denser clustering and more definition along edges. In terms of scene dynamics, higher water levels change the scattering geometry at the edges of the corridors: the surface-volume scattering component is strongly affected as the dry vegetated borders are inundated. A blurring of edges affects only local geometry; the spatial articulation of the main corridors remains unaffected. However, from previously open water can emerge vigorous stands of grasses and aquatic vegetation, shifting areas dominated by Q1 pixels in the December image to Q4 in the May image. The lacunarity index at smaller window sizes measures local heterogeneity and thus should be more sensitive to change in edges; whereas the slope, which measures the articulation of spatial structures across the scene, should remain relatively unaffected. Accordingly, the Q4 lacunarity function shows less change in slope than in values at smaller window sizes (figure $2(d)$ ).

\section{Conclusions}

Lacunarity as a texture measure for.SAR imagery offers several advantages. First, it is a multi-scale technique: dependence of texture on scale of observation can be identified. Second, the decay of the lacunarity index as a function of window size follows characteristic patterns for random, self-similar, and structured spatial arrangements. This feature is especially useful in distinguishing the textural effects of speckle from scene texture. Third, we have demonstrated how lacunarity functions can provide a framework for linking differences in image sequences to changes in scene structure. Lacunarity analysis shows great promise for interpretation and analysis of SAR imagery.

\section{Acknowledgments}

This research was supported through a Fulbright Research Fellowship to G.M.H. and an ERS-1 Pilot Project grant to H.J.H.K. SAR digital imagery was processed by and acquired from the INPE. G.M.H. also acknowledges support from NSF grant BSR-9011662, Long Term Ecological Research at Konza Prairie. Access to the Cray Y-MP4/4-64 at the National Center for Supercomputer Applications, University of Illinois at Urbana-Champaign, was supported by the NCSA under project $t d f$. The authors wish to thank D. Valeriano and two anonymous reviewers for helpful comments.

\section{References}

AlHo, C. J. R., LACHER, JR., T. E., GonçAlVES, H. C., 1988, Environmental degradation in the Pantanal ecosystem. BioScience, 38, 164-171.

BARBER, D. G., and LEDREW, E. F., 1991, SAR sea ice discrimination using texture statistics: a multivariate approach. Photogrammetric Engineering and Remote Sensing, 57, 385-395.

Haralick, R. M., and Shapiro, L. G., 1992, Computer and Robot Vision, Volume 1 (Reading, Massachusetts: Addison-Wesley).

HenebRY, G. M., 1993, Detecting change in grasslands using measures of spatial dependence with Landsat TM data. Remote Sensing of Environment, 46, 223-234.

Karam, M. A., Fung, A. K., Lang, R. H., and Chauhan, N. S., 1992, A microwave scattering model for layered vegetation. I.E.E.E. Transactions on Geoscience and Remote Sensing, 30, 767-784.

KhORos Group, THE, 1991, Khoros Manual, Release 1.0. (Albuquerque, New Mexico: The Khoros Group). 
Mandelbrot, B. B., 1983, The Fractal Geometry of Nature (New York: W. H. Freeman).

PlotNICK, R. E., GARDNer, R. H., and O'NeILL, R. V., 1993, Lacunarity indices as measures of landscape texture. Landscape Ecology, 8, 201-211.

RigNot, E., and KWOK, R., 1993, Characterization of spatial statistics of distributed targets in SAR data. International Journal of Remote Sensing, 14, 345-363.

Rizzinı, C. T., Coimbra Filho, A. F., Houaiss, A., 1988, Ecossitemas Brasileiros/Brazilian Ecosystems (Rio de Janeiro: Index Editora).

SheEn, D. R., and Johnston, L. P., 1992, Statistical and spatial properties of forest clutter measured with polarimetric synthetic aperture radar (SAR). I.E.E.E. Transactions on Geoscience and Remote Sensing, 30, 568-588.

Strahler, A. H., Woodcock, C. E., and SMith, J. A., 1986, On the nature of models in remote sensing. Remote Sensing of Environment, 20, 121-139.

Ulaby, F. T., Kouyate, F., Briscoe, B., and Williams, T. H. L., 1986, Textural information in SAR images. I.E.E.E. Transactions on Geoscience and Remote Sensing, 24, 235-245.

Ulaby, F. T., Saraband, K., McDonald, K., Whitt, M., and Dobson, M. C., 1990, Michigan microwave canopy scattering model. International Journal of Remote Sensing, 11, 1223-1253. 